\title{
Pulsed Thermography: evaluation and quantitative analysis of defects through different post-processing algorithms
}

\author{
by E. D’Accardi, D. Palumbo*, R. Tamborrino*, P. Cavallo*, U. Galietti*
}

*Polytechnic of Bari, Department of mechanics, mathematics and management, Japigia 182, 70126, Bari, ester.daccardi@poliba.it

\begin{abstract}
Pulsed thermography is commonly used as non-destructive technique for evaluating defects within materials and components. However, raw thermal imaging data are usually not suitable for quantitative evaluation of defects. Many data processing algorithms have been developed and each of them provide enhanced detection and sizing of researched defects. In this work, two algorithms have been investigated: Slope, Square of the Correlation Coefficient $\left(\mathrm{R}^{2}\right)$. The aim of this work is to compare these algorithms with the well-established Pulse Phase thermography technique in terms of defects detectability.
\end{abstract}

\section{Introduction}

Pulsed thermography has been used for non-destructive evaluation of different materials and components. As clearly demonstrated in the literature [1-5], the presence of defects reduces the strength of the structure and so, nondestructive tests play a crucial role in the estimation of the useful life of structures and mechanical components.

Compared with other NDE techniques, the thermography is non-contact, fast and easily transportable on site to large and small area inspection. In the case of large components, in fact, it is required a rapid and easy inspection of the same in order to reduce the times and the costs of the ordinary maintenance of the tested components [3-7].

A pulsed thermal experimental test usually consists of a thermal source, with one or more flash lamps, to provide instantaneous heating of the component and an infrared camera to monitor the surface temperature decay for a fixed period of time after the thermal impulse. The defects block the flow of heat from the flash heated surface, causing a reduction in the cooling rate of the surface above the defects that is revealed as an area of thermal contrast in the thermal images of the surface. The temperature contrast between the defected and sounded regions enables defect detection based on thermographic data. Raw thermal data are usually not suitable for quantitative defects evaluation and so, in literature, different algorithms have been proposed to obtain quantitative information from thermographic data [2-4].

In this work the attention has been focused on the Pulsed Thermography technique applied on an aluminum specimen with 20 flat bottom holes of different depths and sizes. Two algorithms have been investigated with the aim to optimize the results in terms of signal to noise ratio and time processing: Square of the Correlation Coefficient $\left(R^{2}\right)$ and Slope [5-6-7]. In particular, the influence of the number of frames on the signal contrast has been considered for each algorithm and a quantitative data analysis has been performed by means a semiautomatic algorithm, capable to discern defected from sounded area [2]. The Pulse Phase Thermography algorithm has been used as comparison [12-14].

\section{Materials and methods}

\subsection{Materials}

An aluminium sample, with 20 flat bottom holes of different diameter and depth, has been testedFigure 1. The different sizes of simulated defects are indicated in Table 1 [2].

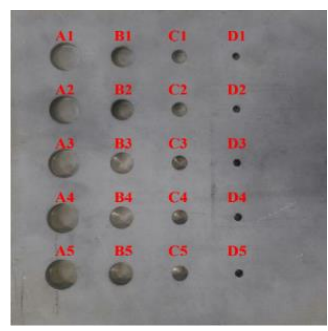

Figure 1: Aluminium sample used for tests. 
Table 1: Sizes of flat bottom holes (aluminium sample in Figure 1).

\begin{tabular}{ccc}
$\begin{array}{c}\text { NUMERATION OF FLAT } \\
\text { BOTTOM HOLES (FIG.2) }\end{array}$ & DIAMETER & DEPTH \\
\hline A1 A2 A3 A4 A5 & A 16mm & A1 1mm A2 2mm A3 3mm A4 4mm A5 5mm \\
B1 B2 B3 B4 B5 & B 12mm & B1 1mm B2 2mm B3 3mm B4 4mm B5 5mm \\
C1 C2 C3 C4 C5 & C 8mm & C1 1mm C2 2mm C3 3mm C4 4mm C 5mm \\
D1 D2 D3 D4 D5 & D 4mm & D1 1mm D2 2mm D3 3mm D4 4mm D5 5mm \\
\hline
\end{tabular}

A pulsed thermography test was performed using the IR camera FLIR X6540 SC with thermal sensitivity (NETD) $<25 \mathrm{mK}$ and based on a cooled detector with $640 \times 512$ pixels. The used set-up is shown in Figure 2. In particular, two flash laps with an energy of $3000 \mathrm{~J}$ were positioned very close to the specimen $(10 \mathrm{~mm})$ and at the same side of the IR camera. This latter was placed at about $1 \mathrm{~m}$ from the specimen in order to obtain a geometrical resolution of $0.25 \mathrm{~mm} / \mathrm{pixel}$.

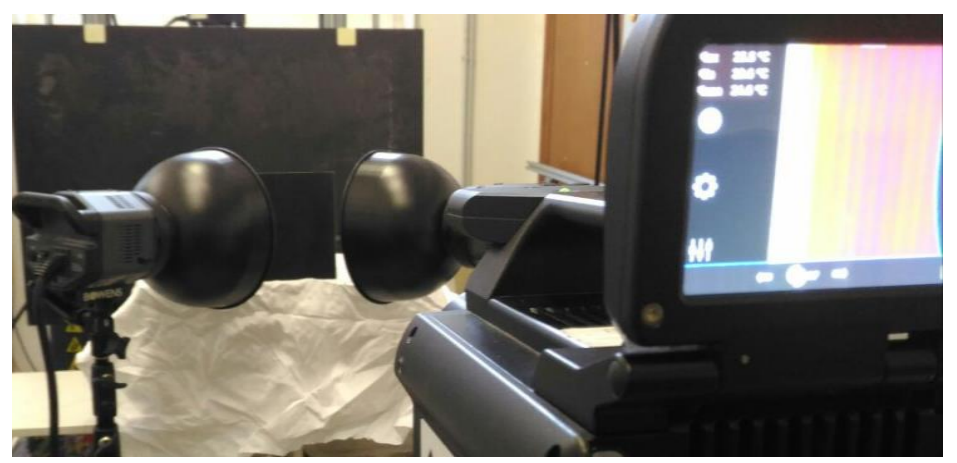

Figure 2: Set-up used for pulsed thermography tests.

The thermal sequence was acquired with a sample rate of $200 \mathrm{~Hz}$, with an observation time of 5 seconds. It is important to underline as $200 \mathrm{~Hz}$ represents the maximum value of the sample rate selectable from IR camera to obtain the full frame of the whole specimen.

\subsection{Methods: Pulsed Infrared Thermography}

The pulsed infrared thermography consists of a short heat pulse to stimulate the surface of tested material; after this thermal heating, the thermal response of the material is analysed. Immediately after the heat pulse, in fact, it is possible to observe the cooling behaviour of tested sample, different between defected and sounded zones. Assuming that the thermal behaviour of the analysed component is the same of a semi-infinite homogenous sample characterized by an effusivity in $z$ direction $e_{z}$ and a homogeneous heating of the same, the surface temperature time evolution follows the law:

$$
\Delta T_{x y}=\frac{Q_{x y}}{e_{z} \sqrt{\pi t}}
$$

where $\Delta T_{x y}$ is the increasing of temperature (in $x$ and $y$ directions), $Q_{x y}$ is the energy absorbed by the surface and $t$ is the time. The temperature decrease rate is different between the defected and sounded zones because there is a difference between the values of the thermal effusivity of these compared regions. The thermal effusivity is defined as:

$$
e_{z}=\sqrt{k \rho c}
$$

where $\mathrm{k}$ is the thermal conductivity, $\rho$ is the mass density and $\mathrm{c}$ is the specific heat of the material. The quantitative measure of the difference between values of the surface temperature referred to sounded and defected regions of the material is the thermal contrasts defined as:

$$
C(t)=\frac{T_{d e f}(t)-T_{d e f}\left(t_{0}\right)}{T_{S}(t)-T_{S}\left(t_{0}\right)}
$$


where Tdef is the temperature of the defected area, Ts is the temperature of the sounded area, to is the time just before pulse heating and $t$ is the current time of the thermal cooling.

In literature, it has been shown that there is a maximum in the trend of the thermal contrast vs cooling time and the time corresponding to this maximum thermal contrast is an index of defect size.

However, in most works, a quantitative analysis is missing to discern the depths and sizes information. In particular, in this work the change of the contrast trend in the time is observed in order to choose the right number of frames to a quantitative analysis of acquired thermographic data. In order to compare the different used algorithms, the contrast, for every tested parameter, is defined as:

$$
C_{n}=\frac{P_{\text {def }}-P_{\text {sound }}}{\sigma}
$$

where $P$ is the chosen parameter and $\sigma$ is the standard deviation. All the calculated contrasts have been normalized respect to standard deviation to consider the noise influence, in order to obtain the so-called noise-signal ratio (SNR) to compare the different algorithms in terms of obtained maximum contrast.

\subsection{Methods: analysed algorithms}

\subsubsection{Pulsed Phase Thermography (PPT)}

Pulsed Phase Thermography is a post-processing algorithm that transforms thermographic data from time domain into frequency domain, using the Discrete Fourier Transform (DFT). The DFT can be derived by discretizing both time and frequency, following the relation [12-14]:

$$
F_{n}=\Delta t \sum_{k=0}^{N-1} T(\Delta t k) \exp ^{\frac{2 j \pi k n}{N}}=R e_{n}+\operatorname{Im}_{n}
$$

where $n$ indicates the frequency increment $(n=0, \ldots, N)$ and $R e$ e $I m$ are the real and the imaginary parts of the transform, respectively. Real and imaginary part of the Eq. 5 can be used to calculate the amplitude and the phase delay of the acquired thermographic sequence, as follows:

$$
A_{n}=\sqrt{R e_{n}^{2}+\operatorname{Im}_{n}^{2}} \quad \Phi_{n}=\tan ^{-1}\left(\frac{I m_{n}}{R e_{n}}\right)
$$

Amplitude and phase are computed by repeating the function for all pixels. Tests parameters should be carefully calibrated according to the material, thickness and depth of the defects that are to be detected.

al noise. In particular, the second derivative sequence provide useful information for the estimation of the defect depths.

\subsubsection{Slope and Square Correlation Coefficient $\mathbf{R}^{2}$}

The Eq. 1 in a double log diagram can be rewritten as [2]:

$$
\ln (\Delta T)=\ln \left(\frac{Q}{e}\right)-\frac{1}{2} \ln (\pi t)
$$

and a representation of this equation is shown in Figure 3 


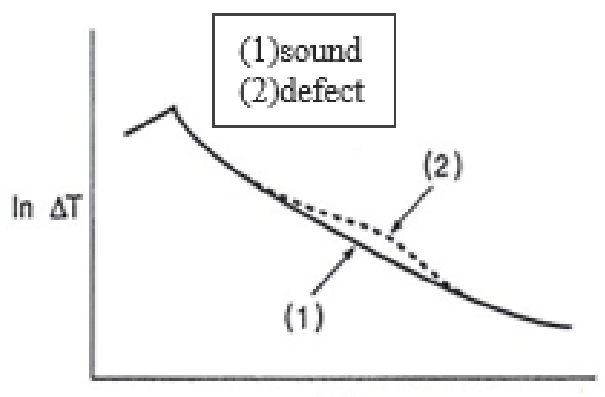

In $t$

Figure 3: Trend of cooling curve in a double logarithmic scale.

If there is a presence of a defect (2), the decay of the temperature variation is different from the one considered in the case of the sounded material and, in general, the slope of this trend is different to $(-1 / 2)$. Furthermore, in the presence of a defect, it is possible to observe a deviation of the cooling trend from a linear one; a measure of this deviation is the square correlation coefficient $R^{2}$. In the work Palumbo D. et al (2016), the algorithms of the slope and the $R^{2}$ have been proposed for the first time to find defects within a composite sample, restoring competitive and comparable results with amplitude and phase maps typically used to analyze thermal data.

\section{Analysis procedure of the acquired data post-processing}

To obtain quantitative information from the acquired data in terms of the signal contrast, it is necessary to provide a post-processing of the same. To reach this aim, the algorithms described in the section 2.3 are computed using MATLAB ${ }^{\circledR}$ software. As anticipated in the Introduction, in literature, there are not indications about the influence of the analyzed number of frames on the quantitative thermographic results. Seven number of frames and then seven intervals of the cooling curve have been chosen for analysis for each algorithm: 16, 32, 64, 128, 256, 512, 1024 frames. The power of 2 for each interval has been chosen to perform a fast post processing of the PPT algorithm (Fast Fourier Transform). The last interval corresponds to 1.28 seconds.

A further data processing is needed to obtain the information about the signal contrast from each algorithm. The procedure used in this work to obtain sounded and defected areas from each defect is the same presented in the work D'Accardi et all [2]. In this case, three tests were carried out keeping the same set-up in order to evaluate the test repeatability.

The steps of the proposed procedure can be summarized as follows:

- Importing of the thermographic sequence (3D matrix);

- Subtracting of the average of the first ten cold frames to the whole sequence to obtain the $\Delta T$ values over time

- $\quad$ Adding an offset $\Delta T$ value to avoid $\Delta T$ values close to zero; this step is applied pixel by pixel to each temperature decay curve and allows to avoid negative values in the logarithmic scale;

- the local temperature values are normalized at any time by dividing them to the values evaluated at time $t$ ' sufficiently near of the pulse occurrence

$$
\Delta \boldsymbol{T}_{x y}(\boldsymbol{t})=\frac{\Delta T_{x y}(t)}{\Delta T_{x y}\left(t^{\prime}\right)}
$$

This pixel-wise operation gives to each pixel, at time $t^{\prime}$, the value equal to 1 . The advantage of this operation is to reduce the effects of non-perfect heating of the sample and the variability of the optical properties of the surface, such as absorptivity and emissivity;

- $\quad$ the 3D final temperature matrix is divided in 7 intervals in order to process the data with the proposed algorithms.

\section{Results}

\subsection{Influence of the number of frames on the signal contrast}

In this section, the influence of the number of frames (size of the analysed time interval) on the signal contrast for each algorithm will be discussed. As already said, seven number of frames corresponding to seven truncation windows over time from $0.08 \mathrm{~s}$ to $5.12 \mathrm{~s}$ were chosen. 
As expected, the number of frames has a great impact on the thermographic results. In this regard, general comments can be done about the results obtained with all algorithms. As it can be seen from the analysis of the graphics for each algorithm (Figure 4, Figure 5, Figure 6), a different behaviour of the thermal contrast as the number of frames increases is observed for the superficial defects. In fact, the defects placed to $1 \mathrm{~mm}$ and $2 \mathrm{~mm}$ from the surface present the higher signal contrast within a time interval of $0.08 \mathrm{~s}$. This behaviour is possible because the thermal waves reach these defects already during the heating phase. In this regard, the thermographic technique can be considered as a stepped test rather than a pulsed one. The deeper defects present a maximum value of the signal contrast in correspondence of a suitable value of the number of frames. In particular, this value changes as function of the depth and size of defects. In other words, each defect owns an optimal truncation window of thermal data.

Results will be reported as graphs in which the signal contrast is reported as function of the size and depth of defects. As expected, for all algorithms it is possible to notice as the signal contrast decreases as the size of the defect decreases and the depth increases. In the following paragraphs, for each analysed algorithm, the different graphs will be shown with the related comments.

\subsubsection{PPT}

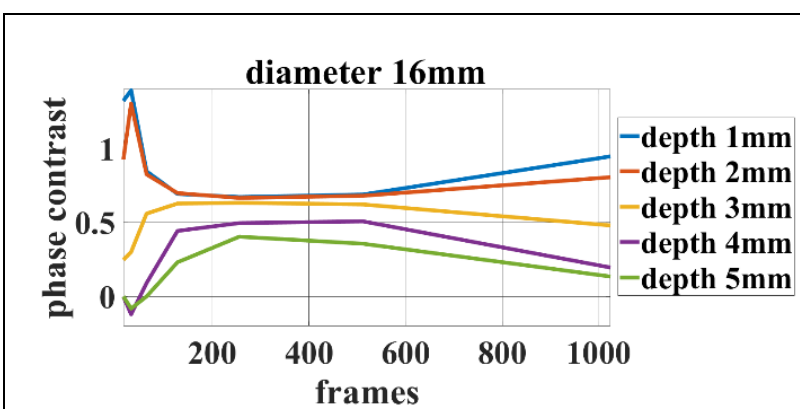

a)

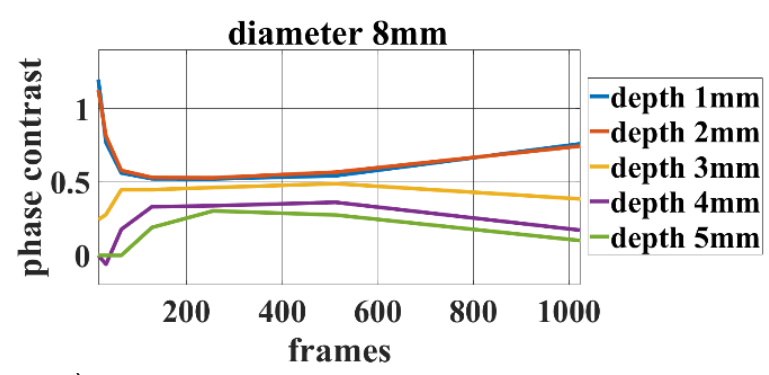

c)

Figure 4: phase contrast vs different frames intervals; a) diameter $16 \mathrm{~mm}$ b) diameter $12 \mathrm{~mm} \mathrm{c}$ ) diameter $8 \mathrm{~mm} \mathrm{~d}$ ) diameter $4 \mathrm{~mm}$ (fixed depth)

In literature, there are very few works that investigate about the importance of the choice of the truncation window on PPT results. In particular, it is suggested [14] that the number of frames $N$ could be further reduced without loss of pertinent information using a high sampling rate. By applying the FFT analysis on the thermographic sequence, a phase map has been obtained for each extracted frequency. For each defect, the trend of phase as a function of the frequency has been analyzed. In particular, the difference of this trend between the defect and the relative sound area shows a peak at the frequency which depends from the depth and the size of the defect. At this frequency, the contrast between the defect and the sound area is maximum. In order to compacting the obtained results, it is chosen to consider the maximum contrast map for each analysis interval regardless of the frequency at which it takes place (D'Accardi et all).

As already said, for the superficial defects ( 1 and $2 \mathrm{~mm}$ of depths, for all sizes), the maximum contrast is obtained analysing an interval of 16 frames. In contrast, the choice to analyse an interval of 1024 frames returns an appreciable signal contrast for the superficial defects, but it seems to decrease for the deeper defects. A good compromise to detect the greatest number of defects with a good contrast is to analyse a number of frames between 256 and 512. 


\subsubsection{Slope}

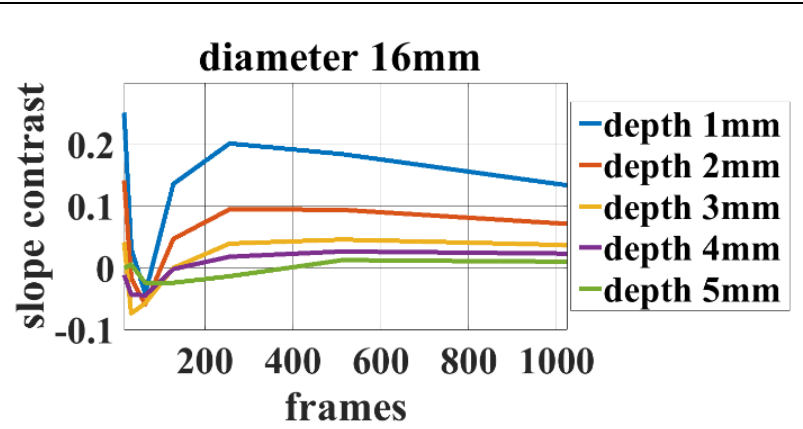

a)

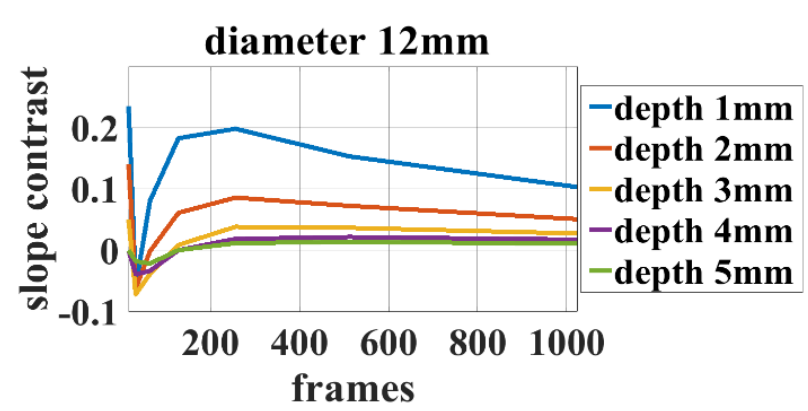

b)

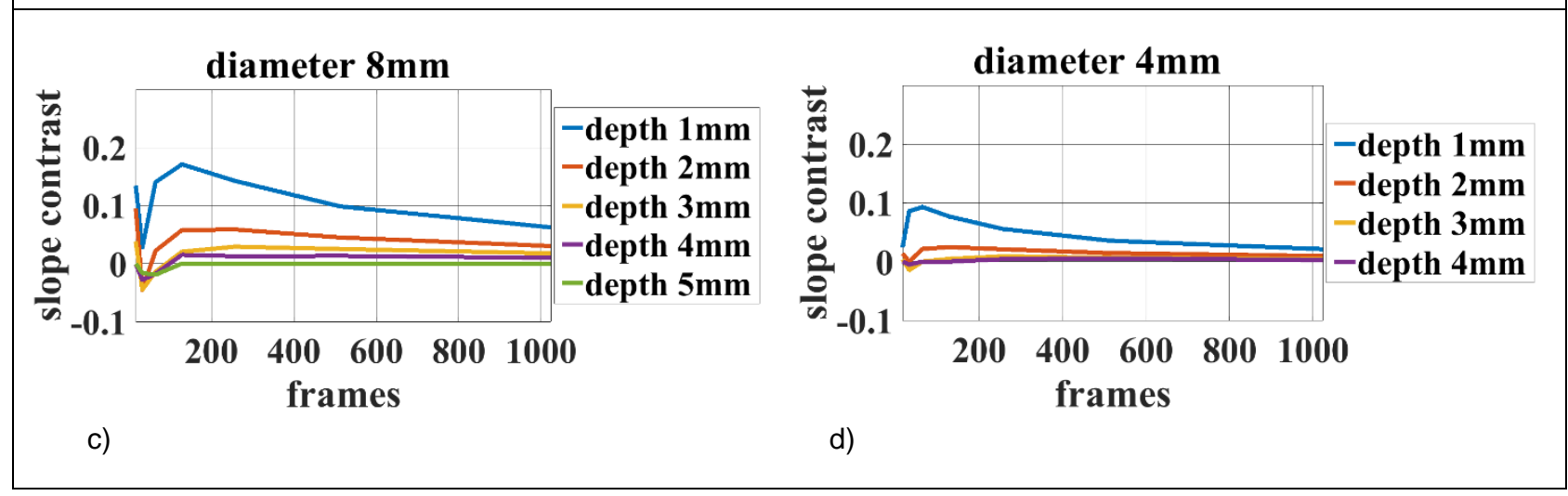

Figure 5: slope contrast vs different frames intervals; a) diameter $16 \mathrm{~mm}$ b) diameter $12 \mathrm{~mm} \mathrm{c}$ ) diameter $8 \mathrm{~mm} \mathrm{d)} \mathrm{diameter}$ $4 \mathrm{~mm}$ (fixed depth)

The trends of the signal contrast for this parameter seem fairly regular. Again, the superficial defects present the maximum contrast in correspondence of few analyzed frames. Defects of 16 and $12 \mathrm{~mm}$ sizes present a change in sign for a fixed number of frames equal to about 128. This "blind value" for the signal contrast seems depend also on the depth of defect. However, it is necessary to process more intervals between 64 and 256 frames to evaluate a possible dependence on the depths. Bigger defects (16 and 12mm sizes) reach a maximum contrast for 256 frames that moving to 128 frames for smaller defects. Finally, the signal contrast decreases until to reach a plateau value in correspondence of 512 and 1024 frames.

\subsection{3 $\mathrm{R}^{2}$}

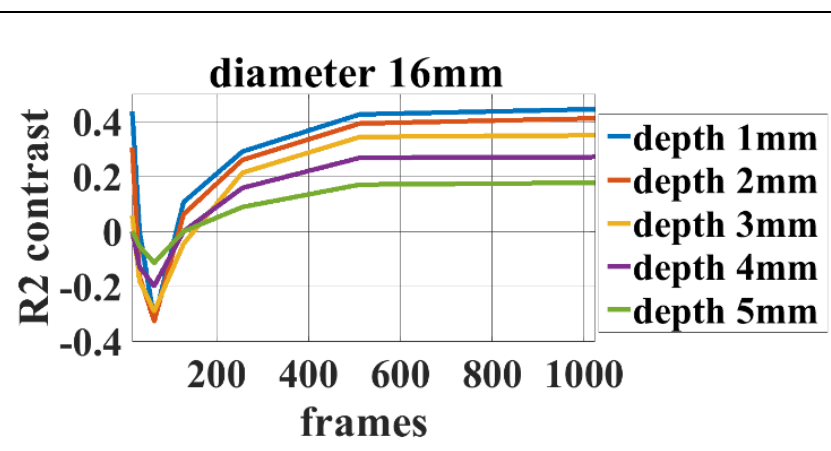

a)

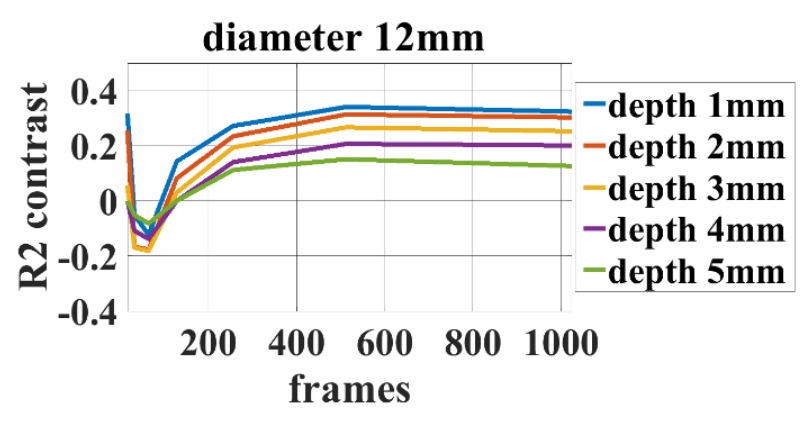

b) 


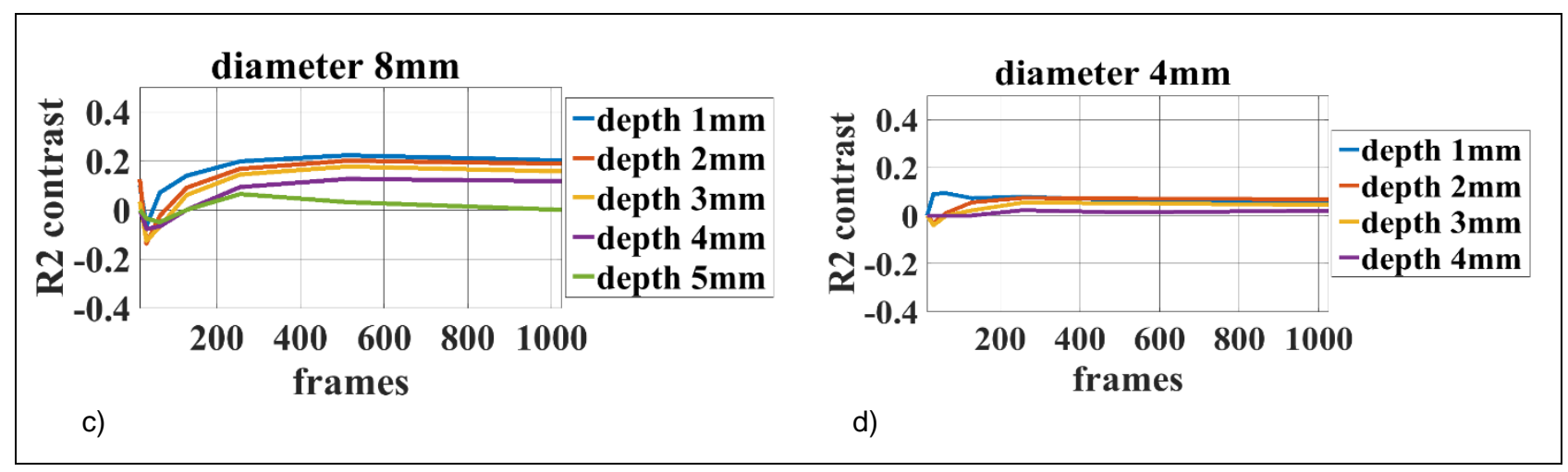

Figure 6: $\mathrm{R}^{2}$ contrast vs different frames intervals; a) diameter $16 \mathrm{~mm}$ b) diameter $12 \mathrm{~mm} \mathrm{c}$ ) diameter $8 \mathrm{~mm}$ d) diameter $4 \mathrm{~mm}$ (fixed depth)

Similar comments can be done about the results obtained analyzing the signal contrast trend of $\mathrm{R}^{2}$. In this case, the maximum contrast for all defects is placed about to 512 frames. In fact, above 512 frames, the signal contrast does not change significantly and then it seems superfluous because the computed times increases, and the contrast remains the same.

\subsection{Comparison between the different algorithms: the SNR versus the $d / p$ ratio.}

The results obtained for each algorithm in terms of maximum signal contrast, in different intervals analysis of the cooling curve (number of frames), have been compared in order to show the difference among the several algorithms implemented. As comparison parameter, the SNR vs d/p ratio has been chosen. In order to obtain the signal to noise ratio, the standard deviation has been evaluated referring to the same sound areas indicated in [2] in the section 3.1 in Fig.9.

It is important to underline as the standard deviation changes with the number of frames and so influences the obtained results with respect to the signal contrast discussed in the previous section, especially for short analysis intervals. The analysis of the obtained graphics shows different results for the three implemented algorithms, both in terms of reached maximum contrast and the number of detected defects. In some obtained graphics, the presence of a regular trend has been highlighted since it can be used for estimating defects size and depth knowing the relationship between the contrast vs the $\mathrm{d} / \mathrm{p}$ ratio. In particular, by analysing an interval of 512 or 1024 frames, a perfect linear trend is obtained from the slope analysis, with a good SNR and the highest number of detected defects equal to 17/20. It should also be highlighted that the trends reported in the following graphics are to be understood as the average of the results obtained by analysing separately the 3 replications of tests. In each graph is also reported a threshold value (horizontal line) placed in correspondence of the SNR value of $2 \sigma\left(2^{*} s t d\right)$. This latter represents the value above of which the defect can be considered detected. The maps of each algorithm are also reported for each value of the number of frame and with the symbol "X" have been indicated the defects below the threshold.

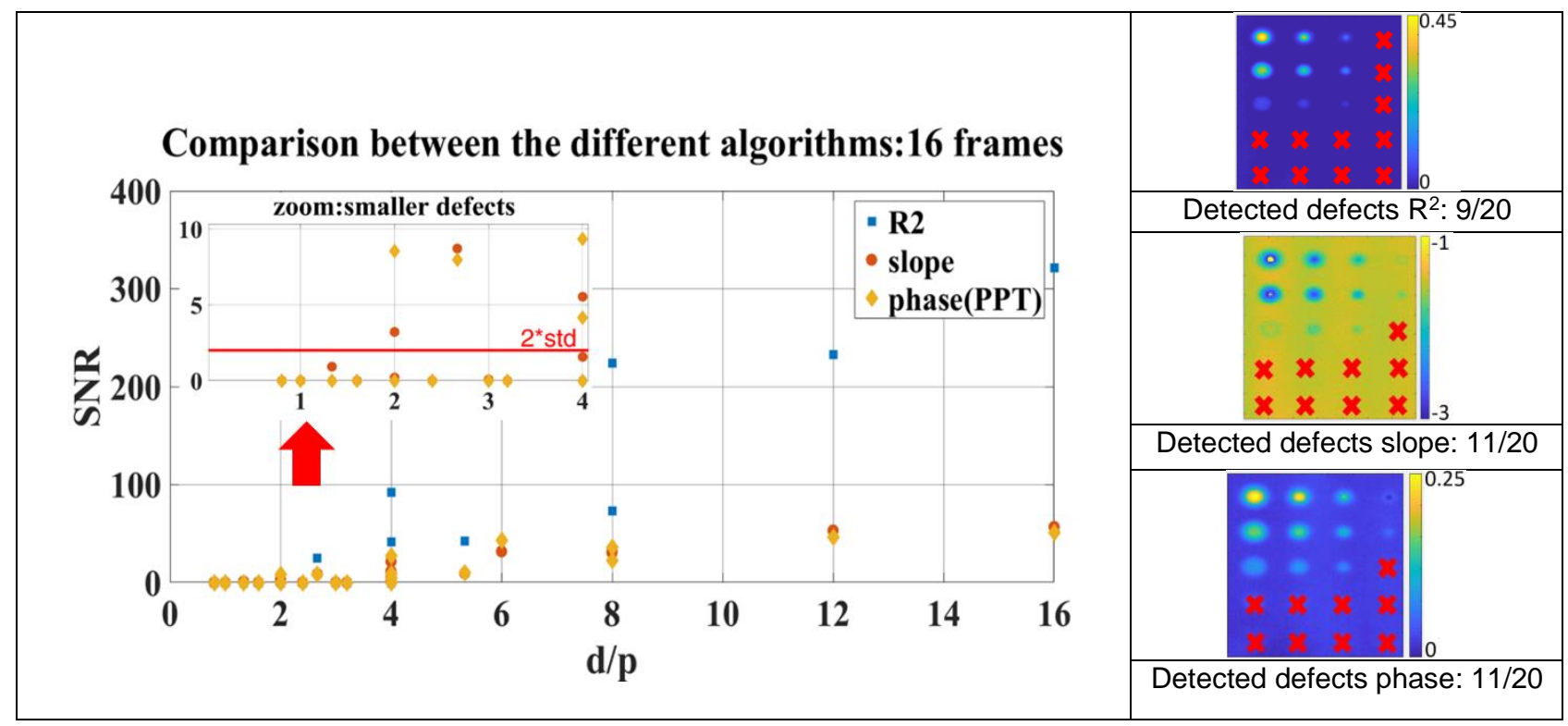




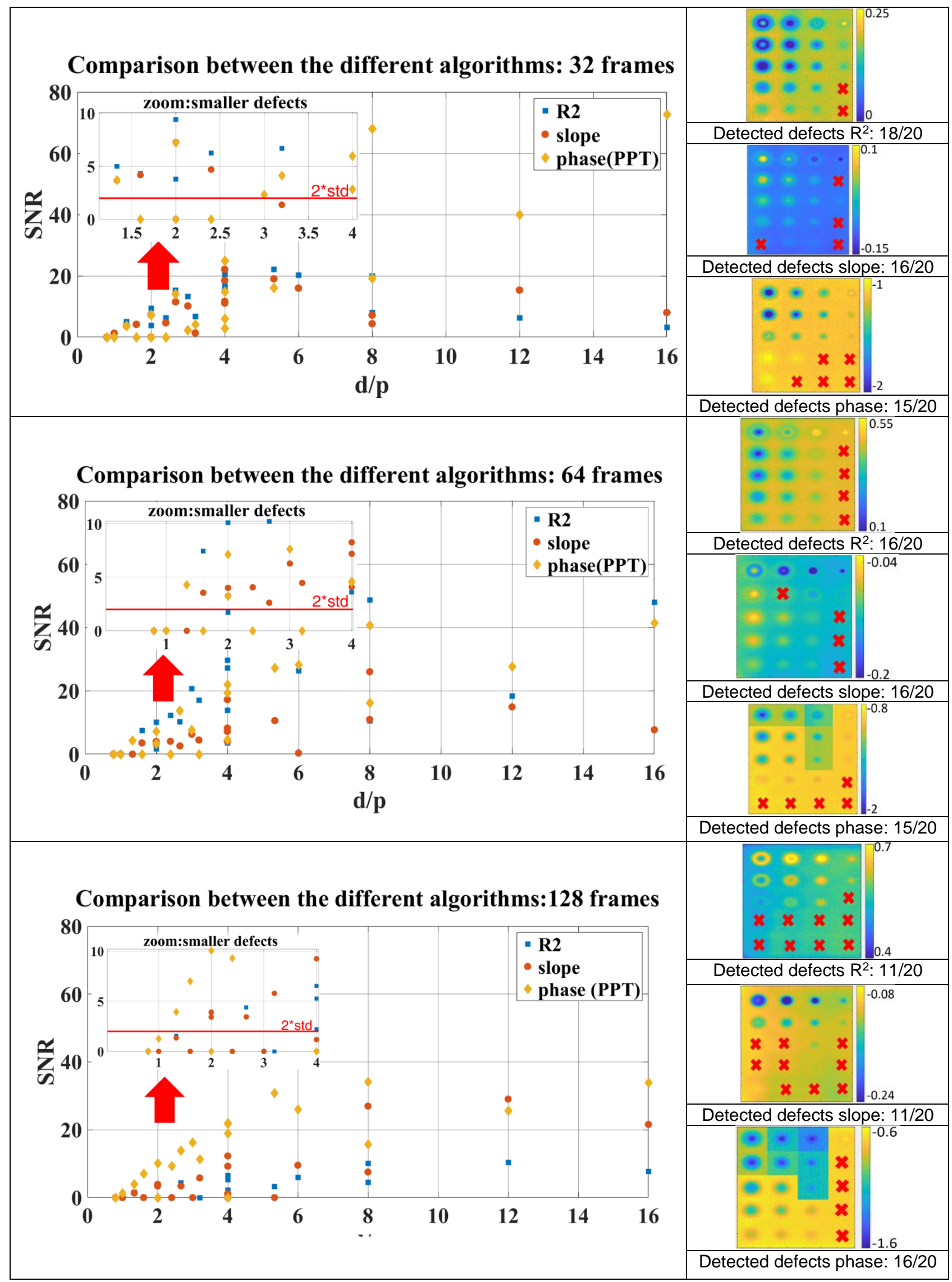




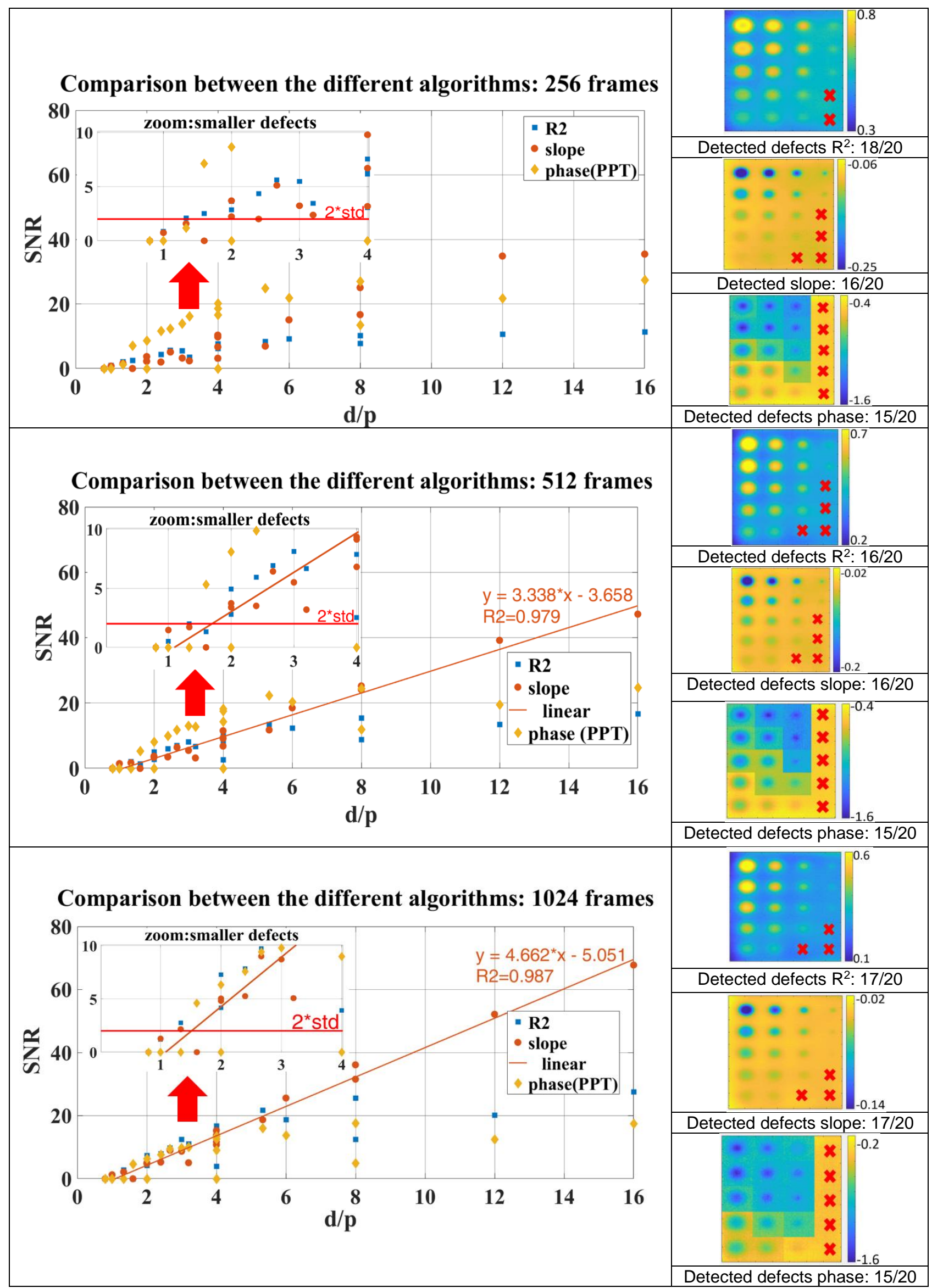




\section{Conclusions}

In this work, two different parameters, slope $(\mathrm{m})$ and $\mathrm{R}^{2}$, obtained by analyzing thermographic data derived by pulsed thermography tests were investigated. PPT algorithm has been used for comparison.

Different tests were carried out on an aluminum specimen with flat bottom holes with the aim to optimize defects detection in terms of testing time and signal to noise ratio. In particular, the number of frames considered for cooling analysis has been taken into account for evaluating the goodness of both parameters.

The main results can be summarized as follow:

- $\quad$ The signal contrast changes with the number of frames chosen for the analysis. In particular, it changes as a function of defects size and depth. Superficial defects $(1$ and $2 \mathrm{~mm})$ present a different trend of the signal contrast vs. the number of frames for each algorithm. These defects give the maximum contrast in correspondence of few considered frames $(16,32,64)$.

- $\quad$ Slope $(\mathrm{m})$ and R2 present a signal contrast with a regular dependence from number of frames and changes in sign in correspondence of a suitable range of number of frames.

- $\quad$ Slope and R2 give the maximum normalized contrast for the higher value of frames $(512,1024)$. However, the R2 allows for detecting 18 defects with few frames (32).

- $\quad$ Phase data (PPT algorithm) present the maximum values of contrast for defects with a $\mathrm{d} / \mathrm{p}<5$.

- $\quad$ The normalized contrast of slope data presents a good correlation (regular linear trend) with the ratio d/p. In this regard, slope parameter can be used to obtain a calibration curve to estimate size and depth of defects.

\section{REFERENCES}

[1] Maldague X. P. V. (2001). Theory and practice of infrared technology of non-destructive testing. John Wiley \& Sons, Inc, ISBN 0-471-18190-0.

[2] E. D'Accardi; D. Palumbo; R. Tamborrino; U. Galietti. (2017). Quantitative analysis of thermographic data through different algorithms. AIAS 2017 International Conference on Stress Analysis, AIAS 2017, Pisa, Italy

[3] Palumbo D., Tamborrino R., Galietti U., Luprano V.A.M. (2016). Ultrasonic analysis and lock-in thermography for debonding evaluation of composite adhesive joints. NDT \& E International Volume 78, Pages 1-9.

[4] Tamborrino R., Palumbo D., Galietti U., Aversa P., Chiozzi S., Luprano V.A.M., (2016). Assessment of the effect of defects on mechanical properties of adhesive bonded joints by using non-destructive methods. Composites Part B, 91, 337-345.

[5] Palumbo D., De Finis R., Demelio G.P., Galietti U. (2016). A new rapid thermographic method to assess the fatigue limit in GFRP composites. Composite Part B, 103, 60-67.

[6] Palumbo D., Galietti U. (2017). Thermal Methods for Evaluating Flaws in Composite Materials: A New Approach to Data Analysis. Mechanics of Composite and Multi-Functional Materials. Volume 7, Proceedings of the 2016 Annual Conference on Experimental and Applied Mechanics, pp. 267-275, ISBN: 978-3-319-41765-3, DOI: 10.1007/978-3-319-41766-0_32.

[7] Galietti U., Palumbo D., Calia G., Pellegrini M. (2012). Non-destructive evaluation of composite materials with thermal methods. 15th European Conference on Composite Materials (ECCM 15), Venice (Italy), ISBN 978-8888785-33-2.

[8] Sun J. (2013). Analysis of data processing methods for pulsed thermal imaging characterisation of delaminations. Quantitative InfraRed Thermography Journal 10(1):9-25

[9] Balageas D.L. (2012). Defense and illustration of time-resolved pulsed thermography for NDE. Quantitative InfraRed Thermography Journal 9:3-32

[10] Hidalgo-Gato R., Andrés J. R., López-Higuera J. M., Madruga F. J. (2013). Quantification by Signal to Noise Ratio of Active Infrared Thermography Data Processing Techniques. Optics and Photonics Journal, 3, 20-26.

[11] Ibarra-Castanedo C., Bendada A. and Maldague X. (2007). Thermographic Image Processing for NDT. IV Conferencia Panamericana de END Buenos Aires.

[12] Ibarra-Castanedo C., Bendada A. and Maldague X. (2005). Image and signal processing techniques in pulsed thermography. GESTS Int'I Trans. Computer Science and Engr., 22(1): 89-100.

[13] Shepard S. M. (2001). Advances in Pulsed Thermography. Proc. SPIE - The International Society for Optical Engineering, Thermosense XXVIII, Orlando, FL, 2001, Eds. A. E. Rozlosnik and R. B. Dinwiddie, 4360:511-515.

[14] Ibarra-Castanedo C. (2005). Quantitative subsurface defect evaluation by pulsed phase thermography: depth retrieval with the phase, Collection Mémories et thèses électroniques.

[15] Palumbo D., Ancona F., Galietti U. (2014). Quantitative damage evaluation of composite materials with microwave thermographic technique: feasibility and new data analysis. Springer Meccanica 50:443-459.

[16] Palumbo D. and Galietti U. (2016). Damage Investigation in Composite Materials by Means of New Thermal Data Processing Procedures. Strain 52(4):276-285

[17] Giorleo G. and Meola C. (2002). Comparison between pulsed and modulated thermography in glass-epoxy laminates. NDT\&E International 35, 287-292.

[18] Balageas D.L., Roche J.M. and Leroy H. (2017). Comparative Assessment of Thermal NDT Data Processing Thecniques for Carbon Fiber Reinforced Polymers, Materials Evaluation 75(8):1019-1031. 